\title{
SCHEDULING WITH POSITION-DEPENDENT WEIGHTS, DUE-DATE ASSIGNMENT AND PAST-SEQUENCE-DEPENDENT SETUP TIMES
}

\author{
Li-Yan Wang ${ }^{1}$, Xue Huang ${ }^{1}$, Wei-Wei Liu ${ }^{2, *}$, Yu-Bin Wu ${ }^{1}$ and Ji-Bo Wang ${ }^{1}$
}

\begin{abstract}
In this study, we consider single-machine scheduling problems with past-sequencedependent (denoted by psd) setup times in which the setup times of jobs are proportional to the length of already processed jobs. Under common (CON) and slack (SLK) due-date assignment methods, we prove that the weighted sum of earliness, tardiness and due-date minimization remains polynomially solvable. We also give some extensions for the scheduling problems with psd setup times.
\end{abstract}

Mathematics Subject Classification. 90B35.

Received March 5, 2020. Accepted October 10, 2020.

\section{INTRODUCTION}

In many realistic scheduling problems, the setup times (costs) of jobs (tasks) are either sequence independent or sequence dependent [1,2]. On the other hand, Koulamas and Kyparisis [16] introduced past-sequencedependent (psd) setup times for single-machine scheduling, i.e., the setup time of a job is dependent on all already scheduled jobs. Using the three-field notation (see [12]), they proved that the scheduling problem $1|\operatorname{psd}| Z$ remains polynomially solvable, where $Z \in\left\{C_{\max }, \sum_{i=1}^{n} C_{i}, \sum_{i=1}^{n} \sum_{j=i}^{n}\left|C_{j}-C_{i}\right|, \lambda \sum_{i=1}^{n} C_{i}+(1-\lambda) \sum_{i=1}^{n} \sum_{j=i}^{n}\left|C_{j}-C_{i}\right|\right\}$, $0 \leq \lambda \leq 1, C_{\max }=\max \left\{C_{i} \mid j=1,2, \ldots, n\right\}$ is the makespan $\left(C_{i}\right.$ is the completion time of job $\left.J_{i}\right), \sum_{i=1}^{n} C_{i}$ is the total completion time, TADC $=\sum_{i=1}^{n} \sum_{j=i}^{n}\left|C_{j}-C_{i}\right|$ is the total absolute differences in completion times. Biskup and Herrmann [4] considered single-machine scheduling problems with psd setup times and due dates. They showed that the problem 1|psd $\mid \sum_{i=1}^{n} L_{i}$ can be solved by the SPT (Smallest Processing Time first) rule, where $L_{i}=C_{i}-d_{i}$ is the lateness of job $J_{i}$, and $d_{i}$ is the due-date of job $J_{i}$. If processing times and due dates are agreeable, they proved that the problem $1|\operatorname{psd}| Z\left(Z \in\left\{\sum_{i=1}^{n} T_{i}, L_{\max }, T_{\max }\right\}\right)$ can be solved in $O(n \log n)$ time, where $T_{i}=\max \left\{0, C_{i}-d_{i}\right\}$ is the tardiness of job $J_{i}, L_{\max }=\max \left\{L_{i}\right\}$ is maximum lateness, and $T_{\max }=\max \left\{T_{i}\right\}$ is maximum tardiness. If a non-restrictive common due-date $d_{\mathrm{opt}}$ is given, Biskup and Herrmann [4] also proved that the problem $1\left|\mathrm{psd}, d_{i}=d_{\mathrm{opt}}\right| Z\left(Z \in\left\{\sum_{i=1}^{n}\left(E_{i}+T_{i}\right), \sum_{i=1}^{n}\left(\alpha E_{i}+\beta T_{i}+\eta d\right), \sum_{i=1}^{n}\left(\alpha E_{i}+\beta T_{i}+\zeta C_{i}\right)\right\}\right)$ can be solved in polynomial time, where $E_{i}=\max \left\{0, d-C_{i}\right\}$ is the earliness of job $J_{i}$, and $\alpha, \beta, \eta, \zeta$ are given constants. Koulamas and Kyparisis [17] proved that the problem $1|\operatorname{psd}| Z\left(Z \in\left\{L_{\max }, T_{\max }, \sum_{i=1}^{n} U_{i}\right\}\right.$, where $U_{i}=1$ if $C_{i}>d_{i}$, otherwise $\left.U_{i}=0\right)$ can be solved in $O\left(n^{2}\right)$ time. They also proposed solution algorithms to solve the problem $1|\operatorname{psd}| Z\left(Z \in\left\{\sum_{i=1}^{n} w_{i} T_{i}, \sum_{i=1}^{n} w_{i} U_{i}, \sum_{i=1}^{n} w_{i}\left(E_{i}+T_{i}\right)\right\}\right)$, where $w_{i}$ is the weight of job $J_{i}$.

Keywords. Scheduling, single-machine, past-sequence-dependent setup times, position-dependent weights.

1 School of Science, Shenyang Aerospace University, Shenyang 110136, P.R. China.

2 Department of Science, Shenyang Sport University, Shenyang 110102, P.R. China.

*Corresponding author: liuww_2010@163.com 
Numerous researchers have considered psd setup times and additional factors such as learning and/or deterioration effects. Cheng et al. [7] investigated single-machine scheduling problems with deteriorating jobs and psd setup times. Kuo and Yang [18], Wang [28], Wang et al. [33], Wang and Wang [31], Kuo et al. [19], Hsu et al. [14], Mani et al. [25], Wang and Li [29], and Soroush [26] examined single-machine scheduling with psd setup times and job-independent (job-dependent) learning effects. Cheng et al. [6], Huang et al. [15], and Wang and Wang [32] studied scheduling problems with deteriorating jobs and learning effects.

For scheduling problems and models, due-date assignment methods have drawn increasing attention $[8,10,11,21,38]$, i.e., jobs are to be completed neither too early nor too late. Brucker [5], Liu et al. [22], and Wang et al. [35] researched single-machine scheduling with due-date assignment and position-dependent weights, i.e., the weight is not related to the job but to the position in which the job is scheduled. Brucker [5] considered the common $(\mathrm{CON})$ due-date assignment with position-dependent weights. Liu et al. [22] dealt with the slack (SLK) due-date assignment with position-dependent weights. Wang et al. [35] scrutinized CON and SLK due-date assignment methods with learning effects and resource allocation. Under position-dependent weights, they proved that several scheduling problems can be solved in polynomial time. "The scheduling problem with psd setup times has many real-world applications. For example, consider the scheduling problem of a high-tech manufacturing environment in which a batch of jobs consisting of a group of electronic components needs to be mounted on an IC board" [16]. This paper extends the results of Brucker [5], Liu et al. [22], and Wang et al. [35], by revisiting psd setup times.

The remaining part of this study is organized as follows. Section 2 formulates the scheduling model. In Sections 3 and 4, we consider CON and SLK due-date assignment problems, respectively. In Section 5, we expound upon the work. Last section presents our conclusions.

\section{Formulation}

Considering a single-machine, on which there are $n$ jobs $J=\left\{J_{1}, J_{2}, \ldots, J_{n}\right\}$ waiting for processing. It is assumed that all the jobs are available at time zero, and preemption (the machine and jobs) is not allowed. Let $s_{i}$ be the psd setup time of job $J_{i}$ and $p_{i}$ be the processing time of job $J_{i}$. We assume that the psd setup time of job $J_{\rho(i)}$ is given as follows:

$$
s_{\rho(1)}=0 \quad \text { and } \quad s_{\rho(i)}=\gamma \sum_{h=1}^{i-1} p_{\rho(h)},
$$

where $\rho(i)$ is some job scheduled in the $i$ th position in a sequence $\rho, \gamma \geq 0$ is a normalizing constant, and total processing requirement of job $J_{\rho(i)}$ is $\gamma \sum_{h=1}^{i-1} p_{\rho(h)}+p_{\rho(i)}$. For a given sequence $\rho$, let $C_{i}=C_{\rho(i)}$ be the completion time of job $J_{i}$, by a mathematical induction, we have

$$
\begin{aligned}
C_{\rho(i)} & =\sum_{j=1}^{i}\left(s_{\rho(j)}+p_{\rho(j)}\right) \\
& =\sum_{j=1}^{i} p_{\rho(j)}+\sum_{j=1}^{i} s_{\rho(j)} \\
& =\sum_{j=1}^{i} p_{\rho(j)}+\sum_{j=1}^{i}\left(\gamma \sum_{h=1}^{j-1} p_{\rho(h)}\right) \\
& =\sum_{j=1}^{i} p_{\rho(j)}+\gamma \sum_{j=1}^{i} \sum_{h=1}^{j-1} p_{\rho(h)}
\end{aligned}
$$




$$
\begin{aligned}
& =\sum_{j=1}^{i} p_{\rho(j)}+\gamma \sum_{j=1}^{i}(i-j) p_{\rho(j)} \\
& =\sum_{j=1}^{i}(1+\gamma(i-j)) p_{\rho(j)} .
\end{aligned}
$$

\section{Common due-DAte Assignment}

For the common $(\mathrm{CON})$ due-date assignment, we have $d_{i}=d_{\mathrm{opt}}, i=1,2, \ldots, n$, where $d_{\mathrm{opt}}$ is a decision variable. The problem is to determine $d_{\mathrm{opt}}$ and a sequence of jobs such that the following total cost is minimized:

$$
\sum_{i=1}^{n} \omega_{i}\left|L_{\rho(i)}\right|+\omega_{0} d_{\mathrm{opt}}=\sum_{i=1}^{n} \omega_{i}\left|C_{\rho(i)}-d_{\mathrm{opt}}\right|+\omega_{0} d_{\mathrm{opt}},
$$

where $\omega_{i}(i=0,1,2, \ldots, n)$ is the non-negative weight of $i$ th position in a sequence (i.e., the position-dependent weights), and $L_{i}=C_{i}-d_{i}$ is lateness of job $J_{i}$. Using the three-field notation (see [12]), the problem can be denoted as $1\left|\mathrm{psd}, \mathrm{CON}, d_{\mathrm{opt}}\right| \sum_{i=1}^{n} \omega_{i}\left|L_{\rho(i)}\right|+\omega_{0} d_{\mathrm{opt}}$, where 1 denotes a single-machine.

Obviously, for an optimal sequence of the problem $1\left|\operatorname{psd}, \mathrm{CON}, d_{\mathrm{opt}}\right| \sum_{i=1}^{n} \omega_{i}\left|L_{\rho(i)}\right|+\omega_{0} d_{\mathrm{opt}}$, there exists no-idle time between the processing of jobs and the first job starts at time zero (see Brucker [5], Lem. 7.1).

Now, we introduce a dummy job $J_{0}$, where its processing time is $p_{0}=0$ and weight is $\omega_{0}$. Obviously, the job $J_{0}$ is always scheduled at time 0 , yielding

$$
\sum_{i=1}^{n} \omega_{i}\left|C_{\rho(i)}-d_{\mathrm{opt}}\right|+\omega_{0} d_{\mathrm{opt}}=\sum_{i=0}^{n} \omega_{i}\left|C_{\rho(i)}-d_{\mathrm{opt}}\right|,
$$

and an optimal sequence is given by $\rho=\left[\rho_{(0)}, \rho_{(1)}, \ldots, \rho_{(n)}\right]$, where $\rho_{(0)}=0$.

Lemma 3.1. For a given sequence $\rho=\left[\rho_{(0)}, \rho_{(1)}, \ldots, \rho_{(n)}\right]$ of the problem $1\left|\mathrm{psd}, \mathrm{CON}, d_{\mathrm{opt}}\right| \sum_{i=1}^{n} \omega_{i}\left|L_{\rho(i)}\right|$ $+\omega_{0} d_{\mathrm{opt}}, d_{\mathrm{opt}}=C_{\rho(k)}=\sum_{i=1}^{k}(1+\gamma(k-i)) p_{\rho(i)}$, where $k$ is a median for the sequence $\omega_{0}, \omega_{1}, \ldots, \omega_{n}$,

$$
\sum_{i=0}^{k-1} \omega_{i} \leq \sum_{i=k}^{n} \omega_{i} \quad \text { and } \quad \sum_{i=0}^{k} \omega_{i} \geq \sum_{i=k+1}^{n} \omega_{i} .
$$

Proof. Let $C_{\rho(k)}<d_{\mathrm{opt}}<C_{\rho(k+1)}$, we have

$$
Z=\sum_{i=1}^{k} \omega_{i}\left(d_{\mathrm{opt}}-C_{\rho(i)}\right)+\sum_{i=k+1}^{n} \omega_{i}\left(C_{\rho(i)}-d_{\mathrm{opt}}\right)+\omega_{0} d_{\mathrm{opt}} .
$$

When $d_{\mathrm{opt}}=C_{\rho(k)}$ and $d_{\mathrm{opt}}=C_{\rho(k+1)}$, we have

$$
\begin{aligned}
Z_{1} & =\sum_{i=1}^{k} \omega_{i}\left(C_{\rho(k)}-C_{\rho(i)}\right)+\sum_{i=k+1}^{n} \omega_{i}\left(C_{\rho(i)}-C_{\rho(k)}\right)+\omega_{0} C_{\rho(k)}, \\
Z_{2} & =\sum_{i=1}^{k+1} \omega_{i}\left(C_{\rho(k+1)}-C_{\rho(i)}\right)+\sum_{i=k+2}^{n} \omega_{i}\left(C_{\rho(i)}-C_{\rho(k+1)}\right)+\omega_{0} C_{\rho(k+1)}, \\
Z-Z_{1} & =\sum_{i=1}^{k} \omega_{i}\left(d_{\mathrm{opt}}-C_{\rho(i)}-C_{\rho(k)}+C_{\rho(i)}\right)+\sum_{i=k+1}^{n} \omega_{i}\left(C_{\rho(i)}-d_{\mathrm{opt}}-C_{\rho(i)}+C_{\rho(k)}\right)+\omega_{0}\left(d_{\mathrm{opt}}-C_{\rho(k)}\right)
\end{aligned}
$$




$$
\begin{aligned}
& =\sum_{i=1}^{k} \omega_{i}\left(d_{\mathrm{opt}}-C_{\rho(k)}\right)+\sum_{i=k+1}^{n} \omega_{i}\left(C_{\rho(k)}-d_{\mathrm{opt}}\right)+\omega_{0}\left(d_{\mathrm{opt}}-C_{\rho(k)}\right) \\
& =\left(\sum_{i=0}^{k} \omega_{i}-\sum_{i=k+1}^{n} \omega_{i}\right)\left(d_{\mathrm{opt}}-C_{\rho(k)}\right)
\end{aligned}
$$

and

$$
\begin{aligned}
Z-Z_{2} & =\sum_{i=1}^{k} \omega_{i}\left(d_{\mathrm{opt}}-C_{\rho(i)}-C_{\rho(k+1)}+C_{\rho(i)}\right)+\sum_{i=k+1}^{n} \omega_{i}\left(C_{\rho(i)}-d_{\mathrm{opt}}-C_{\rho(i)}+C_{\rho(k+1)}\right)+\omega_{0}\left(d_{\mathrm{opt}}-C_{\rho(k+1)}\right) \\
& =\sum_{i=1}^{k} \omega_{i}\left(d_{\mathrm{opt}}-C_{\rho(k+1)}\right)+\sum_{i=k+1}^{n} \omega_{i}\left(C_{\rho(k+1)}-d_{\mathrm{opt}}\right)+\omega_{0}\left(d_{\mathrm{opt}}-C_{\rho(k+1)}\right) \\
& =\left(\sum_{i=0}^{k} \omega_{i}-\sum_{i=k+1}^{n} \omega_{i}\right)\left(d_{\mathrm{opt}}-C_{\rho(k+1)}\right)
\end{aligned}
$$

When $\sum_{i=0}^{k} \omega_{i}-\sum_{i=k+1}^{n} \omega_{i} \geq 0\left(\sum_{i=0}^{k} \omega_{i}-\sum_{i=k+1}^{n} \omega_{i} \leq 0\right)$, we have $Z_{1} \leq Z\left(Z_{2} \leq Z\right)$, then $d_{\mathrm{opt}}=C_{\rho(k)}$ $\left(d_{\mathrm{opt}}=C_{\rho(k+1)}\right)$, i.e., $d_{\mathrm{opt}}$ is equal to the completion time of some job.

From the above analysis, when $d_{\mathrm{opt}}=C_{\rho(k)}$, it follows that $\sum_{i=0}^{k} \omega_{i}-\sum_{i=k+1}^{n} \omega_{i} \geq 0$. When $d_{\mathrm{opt}}=C_{\rho(k+1)}$, we have $\sum_{i=0}^{k} \omega_{i}-\sum_{i=k+1}^{n} \omega_{i} \leq 0$, i.e., if $d_{\mathrm{opt}}=C_{\rho(k)}$, we have $\sum_{i=0}^{k-1} \omega_{i}-\sum_{i=k}^{n} \omega_{i} \leq 0$.

In summary, when $d_{\mathrm{opt}}=C_{\rho(k)}$, we have $\sum_{i=0}^{k-1} \omega_{i} \leq \sum_{i=k}^{n} \omega_{i}$ and $\sum_{i=0}^{k} \omega_{i} \geq \sum_{i=k+1}^{n} \omega_{i}$.

Remark. The properties of Lemma 3.1 is the same as Brucker [5].

Lemma 3.2. For a given sequence $\rho=\left[\rho_{(0)}, \rho_{(1)}, \ldots, \rho_{(n)}\right]$ of the problem $1\left|\mathrm{psd}, \mathrm{CON}, d_{\mathrm{opt}}\right| \sum_{i=1}^{n} \omega_{i}\left|L_{\rho(i)}\right|$ $+\omega_{0} d_{\mathrm{opt}}$, the optimal total cost can be written as:

$$
\sum_{i=1}^{n} \omega_{i}\left|L_{\rho(i)}\right|+\omega_{0} d_{\mathrm{opt}}=\sum_{i=1}^{n} \omega_{i}\left|C_{\rho(i)}-d_{\mathrm{opt}}\right|+\omega_{0} d_{\mathrm{opt}}=\sum_{i=1}^{n} \theta_{i} p_{\rho(i)},
$$

where

$$
\theta_{i}=\left\{\begin{array}{lrl}
\sum_{v=0}^{i-1}(1+\gamma(k-i)) \omega_{v}+\sum_{v=i}^{k} \gamma(k-v) \omega_{v}+\sum_{v=k+1}^{n} \gamma(v-k) \omega_{v}, & \text { for } \quad i=1,2, \ldots, k \\
\sum_{v=i}^{n}(1+(v-k-1) \gamma) \omega_{v}, & \text { for } \quad i=k+1, k+2, \ldots, n .
\end{array}\right.
$$

Proof. From Lemma 3.1 and equation (2.2), we have $d_{\mathrm{opt}}=C_{\rho(k)}$ and $C_{\rho(i)}=\sum_{j=1}^{i}(1+\gamma(i-j)) p_{\rho(j)}$, hence

$$
\begin{aligned}
& \sum_{i=1}^{n} \omega_{i}\left|L_{\rho(i)}\right|+\omega_{0} d_{\mathrm{opt}} \\
& \quad=\omega_{0} C_{\rho(k)}+\sum_{i=1}^{k} \omega_{i}\left(C_{\rho(k)}-C_{\rho(i)}\right)+\sum_{i=k+1}^{n} \omega_{i}\left(C_{\rho(i)}-C_{\rho(k)}\right)
\end{aligned}
$$




$$
\begin{aligned}
= & \sum_{i=0}^{k} \omega_{i} \sum_{v=1}^{i} \gamma(k-i) p_{\rho(v)}+\sum_{i=0}^{k} \omega_{i} \sum_{v=i+1}^{k}(1+\gamma(k-v)) p_{\rho(v)} \\
& +\sum_{i=k+1}^{n} \omega_{i} \sum_{v=1}^{k} \gamma(i-k) p_{\rho(v)}+\sum_{i=k+1}^{n} \omega_{i} \sum_{v=k+1}^{i}(1+\gamma(i-v)) p_{\rho(v)} \\
= & \sum_{v=1}^{k} p_{\rho(v)}\left(\sum_{i=0}^{v-1}(1+\gamma(k-v)) \omega_{i}+\sum_{i=v}^{k} \gamma(k-i) \omega_{i}+\sum_{i=k+1}^{n} \gamma(i-k) \omega_{i}\right) \\
& +\sum_{v=k+1}^{n} p_{\rho(v)}\left(\sum_{i=v}^{n}(1+(i-v) \gamma) \omega_{i}\right) \\
= & \sum_{i=1}^{n} \theta_{i} p_{\rho(i)},
\end{aligned}
$$

where

$$
\theta_{i}=\left\{\begin{array}{lrl}
\sum_{v=0}^{i-1}(1+\gamma(k-i)) \omega_{v}+\sum_{v=i}^{k} \gamma(k-v) \omega_{v}+\sum_{v=k+1}^{n} \gamma(v-k) \omega_{v}, & \text { for } \quad i=1,2, \ldots, k \\
\sum_{v=i}^{n}(1+(v-k-1) \gamma) \omega_{v}, & \text { for } \quad i=k+1, k+2 \ldots, n
\end{array}\right.
$$

Lemma 3.3 ([13]). "The sum of products $\sum_{i=1}^{n} x_{i} y_{i}$ is minimized if sequence $x_{1}, x_{2}, \ldots, x_{n}$ is ordered nondecreasingly and sequence $y_{1}, y_{2}, \ldots, y_{n}$ is ordered nonincreasingly or vice versa, and it is maximized if the sequences are ordered in the same way."

The term (3.3) can be minimized by Lemma 3.3, hence the $1 \mid$ psd, CON, $d_{\mathrm{opt}}\left|\sum_{i=1}^{n} \omega_{i}\right| L_{\rho(i)} \mid+\omega_{0} d_{\mathrm{opt}}$ problem can be solved by the following algorithm:

Algorithm 3.4. Step 1. By Lemma 3.1, calculate $k$.

Step 2. By using Lemma 3.3 (let $x_{i}=p_{i}, y_{i}=\theta_{i}$, see (3.4)) to determine the optimal job sequence.

Step 3. Set $d_{\mathrm{opt}}=C_{\rho(k)}=\sum_{i=1}^{k}(1+\gamma(k-i)) p_{\rho(i)}$.

Theorem 3.5. Algorithm 3.4 solves the problem $1\left|\mathrm{psd}, \mathrm{CON}, d_{\mathrm{opt}}\right| \sum_{i=1}^{n} \omega_{i}\left|L_{\rho(i)}\right|+\omega_{0} d_{\mathrm{opt}}$ in $O(n \log n)$ time.

Proof. The correctness of Algorithm 3.4 follows from Lemmas 3.1-3.3. Steps 1 and 3 can be performed in linear time $O(n)$, and Step 2 requires $O(n \log n)$ time. Thus, the overall computational complexity of Algorithm 3.4 is $O(n \log n)$.

The following example is used to illustrate Algorithm 3.4 for the $1\left|\mathrm{psd}, \mathrm{CON}, d_{\mathrm{opt}}\right| \sum_{i=1}^{n} \omega_{i}\left|L_{\rho(i)}\right|+\omega_{0} d_{\mathrm{opt}}$ problem.

Example 3.6. Consider $n=8, \gamma=0.5$ : the processing times are $p_{1}=7, p_{2}=5, p_{3}=6, p_{4}=9, p_{5}=10$, $p_{6}=3, p_{7}=8, p_{8}=11$; the position-dependent weights are $\omega_{0}=4, \omega_{1}=2, \omega_{2}=3, \omega_{3}=5, \omega_{4}=1, \omega_{5}=8$, $\omega_{6}=7, \omega_{7}=6, \omega_{8}=9$.

By Algorithm 3.4, according to Lemma 3.1, $k=5$. From Lemma 3.2, we have $\theta_{1}=49, \theta_{2}=48$, $\theta_{3}=46.5, \theta_{4}=44.5, \theta_{5}=38, \theta_{6}=34, \theta_{7}=27, \theta_{8}=18$. From Lemma 3.3, the optimal sequence is $\rho=\left[J_{6}, J_{2}, J_{3}, J_{1}, J_{7}, J_{4}, J_{5}, J_{8}\right], d_{\mathrm{opt}}=C_{\rho(5)}=\sum_{j=1}^{5}(1+0.5 *(5-j)) p_{\rho(j)}=52$, and $\sum_{i=1}^{n} \omega_{i}\left|L_{\rho(i)}\right|+$ $\omega_{0} d_{\mathrm{opt}}=2055.5$. 


\section{Slack DUe-DAte Assignment}

For the slack (SLK) due date assignment, we have $d_{i}=s_{i}+p_{i}+q_{\mathrm{opt}}$, where $q_{\mathrm{opt}}$ is a decision variable. The problem is to determine $q_{\text {opt }}$ and a sequence of jobs such that the following cost is minimized:

$$
\sum_{i=1}^{n} \omega_{i}\left|L_{\rho(i)}\right|+\omega_{0} q_{\mathrm{opt}}=\sum_{i=1}^{n} \omega_{i}\left|C_{\rho(i)}-d_{\rho(i)}\right|+\omega_{0} q_{\mathrm{opt}} .
$$

Using the three-field notation, the problem can be denoted as $1 \mid \operatorname{psd}$, SLK, $q_{\mathrm{opt}}\left|\sum_{i=1}^{n} \omega_{i}\right| L_{\rho(i)} \mid+\omega_{0} q_{\mathrm{opt}}$.

Obviously, for an optimal sequence of the problem $1\left|\mathrm{psd}, \mathrm{SLK}, q_{\mathrm{opt}}\right| \sum_{i=1}^{n} \omega_{i}\left|L_{\rho(i)}\right|+\omega_{0} q_{\mathrm{opt}}$, there exists no-idle time between the processing of jobs, and the first job starts at time zero (see [22]).

Similar to Section 3 , we introduce a dummy job $J_{0}$ (its processing time $p_{0}=0$ and weight $\omega_{0}$ ) which is always scheduled at time 0 , then

$$
\sum_{i=1}^{n} \omega_{i}\left|C_{\rho(i)}-d_{\rho(i)}\right|+\omega_{0} q_{\mathrm{opt}}=\sum_{i=0}^{n} \omega_{i}\left|C_{\rho(i)}-d_{\rho(i)}\right|,
$$

and an optimal sequence is given by $\rho=\left[\rho_{(0)}, \rho_{(1)}, \ldots, \rho_{(n)}\right]$, where $\rho_{(0)}=0$.

Lemma 4.1. If $C_{\rho(i)} \geq d_{\rho(i)}$ then $C_{\rho(i+1)} \geq d_{\rho(i+1)}$, and if $C_{\rho(i)} \leq d_{\rho(i)}$ then $C_{\rho(i-1)} \leq d_{\rho(i-1)}$.

Proof. If $C_{\rho(i)} \geq d_{\rho(i)}=s_{\rho(i)}+p_{\rho(i)}+q_{\mathrm{opt}} \geq q_{\mathrm{opt}}$, then $C_{\rho(i+1)}=C_{\rho(i)}+s_{\rho(i)}+p_{\rho(i)} \geq d_{\rho(i)}+s_{\rho(i)}+p_{\rho(i)} \geq$ $q_{\mathrm{opt}}+s_{\rho(i)}+p_{\rho(i)}=d_{\rho(i+1)}$.

If $C_{\rho(i)} \leq d_{\rho(i)}$, then $C_{\rho(i-1)}+s_{\rho(i)}+p_{\rho(i)} \leq s_{\rho(i)}+p_{\rho(i)}+q_{\mathrm{opt}}, C_{\rho(i-1)} \leq q_{\mathrm{opt}} \leq s_{\rho(i-1)}+p_{\rho(i-1)}+q_{\mathrm{opt}}=$ $d_{\rho(i-1)}$.

Lemma 4.2. For a given sequence $\rho=\left[\rho_{(0)}, \rho_{(1)}, \ldots, \rho_{(n)}\right]$ of the problem $1\left|\operatorname{psd}, \mathrm{SLK}, q_{\mathrm{opt}}\right| \sum_{i=1}^{n} \omega_{i}\left|L_{\rho(i)}\right|$ $+\omega_{0} q_{\mathrm{opt}}, q_{\mathrm{opt}}=C_{\rho(l)}=\sum_{i=1}^{l}(1+\gamma(l-i)) p_{\rho(i)}$, where $l$ is a median for the sequence $\omega_{0}, \omega_{1}, \ldots, \omega_{n}$,

$$
\sum_{i=0}^{l} \omega_{i} \leq \sum_{i=l+1}^{n} \omega_{i} \quad \text { and } \quad \sum_{i=0}^{l+1} \omega_{i} \geq \sum_{i=l+2}^{n} \omega_{i}
$$

Proof. Define $C_{\rho(l)}<q_{\mathrm{opt}}<C_{\rho(l+1)}$, then $C_{\rho(l)}+s_{\rho(l+1)}+p_{\rho(l+1)}<q_{\mathrm{opt}}+s_{\rho(l+1)}+p_{\rho(l+1)}<C_{\rho(l+1)}+s_{\rho(l+1)}+$ $p_{\rho(l+1)}$, we have $C_{\rho(l+1)}<d_{\rho(l+1)}$. Since $C_{\rho(l)}+s_{\rho(l+2)}+p_{\rho(l+2)}<q_{\mathrm{opt}}+s_{\rho(l+2)}+p_{\rho(l+2)}<C_{\rho(l+1)}+s_{\rho(l+2)}+$ $p_{\rho(l+2)}$, it follows that $d_{\rho(l+2)}<C_{\rho(l+2)}$. From Lemma 4.1, we have

$$
\begin{aligned}
Z & =\sum_{i=1}^{l+1} \omega_{i}\left(d_{\rho(i)}-C_{\rho(i)}\right)+\sum_{i=l+2}^{n} \omega_{i}\left(C_{\rho(i)}-d_{\rho(i)}\right)+\omega_{0} q_{\mathrm{opt}} \\
& =\sum_{i=1}^{l+1} \omega_{i}\left(s_{\rho(i)}+p_{\rho(i)}+q_{\mathrm{opt}}-C_{\rho(i)}\right)+\sum_{i=l+2}^{n} \omega_{i}\left(C_{\rho(i)}-s_{\rho(i)}-p_{\rho(i)}-q_{\mathrm{opt}}\right)+\omega_{0} q_{\mathrm{opt}} .
\end{aligned}
$$

When $q_{\text {opt }}=C_{\rho(l)}$, then $d_{\rho(i)}=s_{\sigma(i)}+p_{\sigma(i)}+C_{\rho(l)}$,

$$
Z_{1}=\sum_{i=1}^{l+1} \omega_{i}\left(s_{\rho(i)}+p_{\rho(i)}+C_{\rho(l)}-C_{\rho(i)}\right)+\sum_{i=l+2}^{n} \omega_{i}\left(C_{\rho(i)}-s_{\rho(i)}-p_{\rho(i)}-C_{\rho(l)}\right)+\omega_{0} C_{\rho(l)} .
$$

When $q_{\mathrm{opt}}=C_{\rho(l+1)}$, then $d_{\rho(i)}=s_{\rho(i)}+p_{\rho(i)}+C_{\rho(l+1)}$,

$$
Z_{2}=\sum_{i=1}^{l+1} \omega_{i}\left(s_{\rho(i)}+p_{\rho(i)}+C_{\rho(l+1)}-C_{\rho(i)}\right)+\sum_{i=l+2}^{n} \omega_{i}\left(C_{\rho(i)}-s_{\rho(i)}-p_{\rho(i)}-C_{\rho(l+1)}\right)+\omega_{0} C_{\rho(l+1)} .
$$




$$
\begin{aligned}
Z-Z_{1} & =\sum_{i=1}^{l+1} \omega_{i}\left(q_{\mathrm{opt}}-C_{\rho(l)}\right)+\sum_{i=l+2}^{n} \omega_{i}\left(C_{\rho(l)}-q_{\mathrm{opt}}\right)+\omega_{0}\left(q_{\mathrm{opt}}-C_{\rho(l)}\right) \\
& =\left(\sum_{i=0}^{l+1} \omega_{i}-\sum_{i=l+2}^{n} \omega_{i}\right)\left(q_{\mathrm{opt}}-C_{\rho(l)}\right), \\
Z-Z_{2} & =\sum_{i=1}^{l+1} \omega_{i}\left(q_{\mathrm{opt}}-C_{\sigma(l+1)}\right)+\sum_{i=l+2}^{n} \omega_{i}\left(C_{\rho(l+1)}-q_{\mathrm{opt}}\right)+\omega_{0}\left(q_{\mathrm{opt}}-C_{\sigma(l+1)}\right) \\
& =\left(\sum_{i=0}^{l+1} \omega_{i}-\sum_{i=l+2}^{n} \omega_{i}\right)\left(q_{\mathrm{opt}}-C_{\rho(l+1)}\right) .
\end{aligned}
$$

When $\sum_{i=0}^{l+1} \omega_{i}-\sum_{i=l+2}^{n} \omega_{i} \geq 0\left(\sum_{i=0}^{l+1} \omega_{i}-\sum_{i=l+2}^{n} \omega_{i} \leq 0\right), Z_{1} \leq Z\left(Z_{2} \leq Z\right)$, then $q_{\mathrm{opt}}=C_{\rho(l)}\left(q_{\mathrm{opt}}=C_{\rho(l+1)}\right)$, i.e., $q_{\mathrm{opt}}$ is equal to the completion time of some job.

From the above analysis, when $q_{\mathrm{opt}}=C_{\rho(l)}$, it follows that $\sum_{i=0}^{l+1} \omega_{i}-\sum_{i=l+2}^{n} \omega_{i} \geq 0$. When $q_{\mathrm{opt}}=C_{\rho(l+1)}$, it follows that $\sum_{i=0}^{l+1} \omega_{i}-\sum_{i=l+2}^{n} \omega_{i} \leq 0$, so, when $q_{\text {opt }}=C_{\rho(l)}$, it follows that $\sum_{i=0}^{l} \omega_{i}-\sum_{i=l+1}^{n} \omega_{i} \leq 0$.

In summary, when $q_{\mathrm{opt}}=C_{\rho(l)}$, we have $\sum_{i=0}^{l} \omega_{i} \leq \sum_{i=l+1}^{n} \omega_{i}$ and $\sum_{i=0}^{l+1} \omega_{i} \geq \sum_{i=l+2}^{n} \omega_{i}$.

Remark. The properties of Lemmas 4.1 and 4.2 is the same as Liu et al. [22].

Lemma 4.3. For the problem $1\left|\mathrm{psd}, \mathrm{SLK}, q_{\mathrm{opt}}\right| \sum_{i=1}^{n} \omega_{i}\left|L_{\rho(i)}\right|+\omega_{0} q_{\mathrm{opt}}$, the optimal total cost can be written as:

$$
\sum_{i=1}^{n} \omega_{i}\left|L_{\rho(i)}\right|+\omega_{0} q_{\mathrm{opt}}=\sum_{i=1}^{n} \omega_{i}\left|C_{\rho(i)}-d_{\rho(i)}\right|+\omega_{0} q_{\mathrm{opt}}=\sum_{i=1}^{n} \theta_{i} p_{\rho(i)}
$$

where

$$
\theta_{i}= \begin{cases}\sum_{v=0}^{i}(1+\gamma(l-i)) \omega_{v}+\sum_{v=i+1}^{l+1} \gamma(l-v+1) \omega_{v} & \\ +\sum_{v=l+2}^{n} \gamma(v-l-1) \omega_{v}, & \text { for } \quad i=1,2, \ldots, l \\ \sum_{v=i+1}^{n}(1+(v-l-2) \gamma) \omega_{v}, & \text { for } \quad i=l+1, l+2, \ldots, n-1 ; \\ 0, & \text { for } \quad i=n .\end{cases}
$$

Proof. Let $\rho=\left[\rho_{(0)}, \rho_{(1)}, \ldots, \rho_{(n)}\right]$ and $q_{\mathrm{opt}}=C_{\sigma(l)}$ be an optimal solution such that equation (4.2) can be satisfied, we have

$$
\begin{aligned}
\sum_{i=1}^{n} \omega_{i}\left|L_{\rho(i)}\right|+\omega_{0} q_{\mathrm{opt}} & \\
= & \omega_{0} C_{\rho(l)}+\sum_{i=1}^{l+1} \omega_{i}\left(C_{\rho(l)}-C_{\rho(i)}\right)+\sum_{i=l+2}^{n} \omega_{i}\left(C_{\rho(i)}-C_{\rho(l)}\right) \\
& =\sum_{i=0}^{l+1} \omega_{i} \sum_{v=1}^{i-1} \gamma(l-i+1) p_{\rho(v)}+\sum_{i=0}^{l+1} \omega_{i} \sum_{v=i}^{l}(1+\gamma(l-v)) p_{\rho(v)}
\end{aligned}
$$




$$
\begin{aligned}
& +\sum_{i=l+2}^{n} \omega_{i} \sum_{v=1}^{l} \gamma(i-l-1) p_{\rho(v)}+\sum_{i=l+2}^{n} \omega_{i} \sum_{v=l+1}^{i-1}(1+\gamma(i-v-1)) p_{\rho(v)} \\
= & \sum_{v=1}^{l} p_{\rho(v)}\left(\sum_{i=0}^{v}(1+\gamma(l-v)) \omega_{i}+\sum_{i=v+1}^{l+1} \gamma(l-i+1) \omega_{i}+\sum_{i=l+2}^{n} \gamma(i-l-1) \omega_{i}\right) \\
& +\sum_{v=l+1}^{n-1} p_{\rho(v)}\left(\sum_{i=v+1}^{n}(1+(i-v-1) \gamma) \omega_{i}\right) \\
= & \sum_{i=1}^{n} \theta_{i} p_{\rho(i)},
\end{aligned}
$$

where

$$
\theta_{i}= \begin{cases}\sum_{v=0}^{i}(1+\gamma(l-i)) \omega_{v}+\sum_{v=i+1}^{l+1} \gamma(l-v+1) \omega_{v} & \\ +\sum_{v=l+2}^{n} \gamma(v-l-1) \omega_{v}, & \text { for } \quad i=1,2, \ldots, l \\ \sum_{v=i+1}^{n}(1+(v-l-2) \gamma) \omega_{v}, & \text { for } \quad i=l+1, l+2, \ldots, n-1 ; \\ 0, & \text { for } \quad i=n .\end{cases}
$$

The term (4.3) can be minimized by Lemma 3.3; hence the $1 \mid$ psd, SLK, $q_{\text {opt }}\left|\sum_{i=1}^{n} \omega_{i}\right| L_{\rho(i)} \mid+\omega_{0} q_{\text {opt }}$ problem can be solved by the following algorithm:

Algorithm 4.4. Step 1. By Lemma 4.2, calculate $l$.

Step 2. By using Lemma 3.3 (let $x_{i}=p_{i}, y_{i}=\theta_{i}$ (see (4.4))) to determine the optimal job sequence.

Step 3. Set $q_{\mathrm{opt}}=C_{\rho(l)}=\sum_{i=1}^{l}(1+\gamma(l-i)) p_{\rho(i)}$.

Theorem 4.5. Algorithm 4.4 solves the problem $1\left|\operatorname{psd}, \mathrm{SLK}, q_{\mathrm{opt}}\right| \sum_{i=1}^{n} \omega_{i}\left|L_{\rho(i)}\right|+\omega_{0} q_{\mathrm{opt}}$ in $O(n \log n)$ time.

Proof. Similar to the proof of Theorem 3.5.

The following example is used to illustrate Algorithm 4.4 for the problem $1 \mid$ psd, SLK, $q_{\mathrm{opt}}\left|\sum_{i=1}^{n} \omega_{i}\right| L_{\rho(i)} \mid+$ $\omega_{0} q_{\mathrm{opt}}$.

Example 4.6. The input data in this example is the same as in Example 3.6.

By Algorithm 4.4, according to Lemma 4.2, $l=4$. From Lemma 4.3, we have $\theta_{1}=48, \theta_{2}=46.5$, $\theta_{3}=44.5, \theta_{4}=38, \theta_{5}=34, \theta_{6}=27, \theta_{7}=18, \theta_{8}=0$. From Lemma 3.3, the optimal sequence is $\rho=\left[J_{6}, J_{2}, J_{3}, J_{1}, J_{7}, J_{4}, J_{5}, J_{8}\right], q_{\mathrm{opt}}=C_{\rho(4)}=\sum_{j=1}^{4}(1+0.5 *(4-j)) p_{\rho(j)}=33.5$, and $\sum_{i=1}^{n} \omega_{i}\left|L_{\rho(i)}\right|+$ $\omega_{0} q_{\mathrm{opt}}=1604.5$.

\section{Extensions}

\subsection{Truncated job-dependent learning effect}

In this subsection, the proposed model is extended by the introduction of truncated job-dependent learning effect $[3,23,24,27,34,36,37]$, i.e., if job $J_{i}$ is scheduled in the $r$ th position in a sequence, its actual processing time is given by

$$
p_{i}^{A}=p_{i} \max \left\{r^{a_{i}}, b\right\}, i, r=1, \ldots, n,
$$


where $a_{i} \leq 0$ is the job-dependent learning effect, and $b$ is a truncation parameter $(0<b<1)$. The psd setup time of job $J_{\rho(i)}$ is $s_{\rho(i)}=\gamma \sum_{h=1}^{i-1} p_{\rho(h)}^{A}$. For the SLK due-date assignment, $d_{i}=s_{i}+p_{i}^{A}+q_{\mathrm{opt}}$.

Obviously, Lemmas 3.1, 3.2, 4.1 and 4.2 still hold when truncated job-dependent learning effect is introduced. Similar to the above analysis, we have

$$
\sum_{i=1}^{n} \omega_{i}\left|L_{\rho(i)}\right|+\omega_{0} d_{\mathrm{opt}} / q_{\mathrm{opt}}=\sum_{i=1}^{n} \theta_{i} p_{\rho(i)}^{A}=\sum_{i=1}^{n} \theta_{i} p_{\rho(i)} \max \left\{r^{a_{\rho(i)}}, b\right\},
$$

where, for the CON due-date assignment, $\theta_{i}(i=1,2, \ldots, n)$ is given by $(3.4)$; for the SLK due-date assignment, $\theta_{i}(i=1,2, \ldots, n)$ is given by $(4.4)$.

Let

$$
x_{i r}= \begin{cases}1, & \text { if job } J_{i} \text { is assigned to the } r \text { th position, } \\ 0, & \text { otherwise. }\end{cases}
$$

Then, we can formulate the sequence of the problem $1\left|\mathrm{psd}, \mathrm{CON} / \mathrm{SLK}, d_{\mathrm{opt}} / q_{\mathrm{opt}}\right| \sum_{i=1}^{n} \omega_{i}\left|L_{\rho(i)}\right|+\omega_{0} d_{\mathrm{opt}} / q_{\mathrm{opt}}$ as the following assignment problem:

$$
\begin{aligned}
& \operatorname{Min} \sum_{i=1}^{n} \sum_{r=1}^{n} \theta_{r} p_{i} \max \left\{r^{a_{i}}, b\right\} x_{i r} \\
& \text { s.t. } \sum_{i=1}^{n} x_{i r}=1, r=1, \ldots, n \\
& \sum_{r=1}^{n} x_{i r}=1, i=1, \ldots, n \\
& x_{i r}=\{0,1\} .
\end{aligned}
$$

Based on the above analysis, the problem $1\left|\operatorname{psd}, \mathrm{CON} / \mathrm{SLK}, d_{\mathrm{opt}} / q_{\mathrm{opt}}, p_{i}^{A}=p_{i} \max \left\{r^{a_{i}}, b\right\}\right| \sum_{i=1}^{n} \omega_{i}\left|L_{\rho(i)}\right|+$ $\omega_{0} d_{\mathrm{opt}} / q_{\mathrm{opt}}$ can be solved by the following algorithm:

Algorithm 5.1. Step 1. For the CON due-date assignment, by using Lemma 3.1, calculate $k$; For the SLK due-date assignment, by using Lemma 4.2, calculate $l$.

Step 2. Solve the assignment problem (5.3) to (5.6) to determine the optimal sequence.

Step 3. Calculate $d_{\mathrm{opt}}=C_{\rho(k)}=\sum_{i=1}^{k}(1+\gamma(k-i)) p_{\rho(i)}^{A}, q_{\mathrm{opt}}=C_{\rho(l)}=\sum_{i=1}^{l}(1+\gamma(l-i)) p_{\rho(i)}^{A}$.

Based on the above analysis, we have

Theorem 5.2. The problem $1\left|\mathrm{psd}, \mathrm{CON} / \mathrm{SLK}, d_{\mathrm{opt}} / q_{\mathrm{opt}}, p_{i}^{A}=p_{i} \max \left\{r^{a_{i}}, b\right\}\right| \sum_{i=1}^{n} \omega_{i}\left|L_{\rho(i)}\right|+\omega_{0} d_{\mathrm{opt}} / q_{\mathrm{opt}}$ can be solved by Algorithm 5.1 in $O\left(n^{3}\right)$ time.

The following example is only used to illustrate Algorithm 5.1 for the problem $1\left|\mathrm{psd}, \mathrm{CON}, d_{\mathrm{opt}}, p_{i}^{A}=p_{i} \max \left\{r^{a_{i}}, b\right\}\right| \sum_{i=1}^{n} \omega_{i}\left|L_{\rho(i)}\right|+\omega_{0} d_{\mathrm{opt}}$.

Example 5.3. Consider $n=8, \gamma=0.5, b=0.6$ : the processing times are $p_{1}=7, p_{2}=5, p_{3}=6, p_{4}=9$, $p_{5}=10, p_{6}=3, p_{7}=8, p_{8}=11$; the position-dependent weights are $\omega_{0}=4, \omega_{1}=2, \omega_{2}=3, \omega_{3}=5, \omega_{4}=1$, $\omega_{5}=8, \omega_{6}=7, \omega_{7}=6, \omega_{8}=9$ and job-dependent learning effects are $a_{1}=-0.27, a_{2}=-0.25, a_{3}=-0.3$, $a_{4}=-0.29, a_{5}=-0.32, a_{6}=-0.33, a_{7}=-0.28, a_{8}=-0.31$.

By Algorithm 5.1 and Example 3.6, we have $k=5$, and $\theta_{1}=49, \theta_{2}=48, \theta_{3}=46.5, \theta_{4}=44.5, \theta_{5}=38$, $\theta_{6}=34, \theta_{7}=27, \theta_{8}=18$. The values $\theta_{r} p_{i} \max \left\{r^{a_{i}}, b\right\}$ are given in Table 1 . The costs of solution for the assignment problem (5.3-5.6) are given in bold in Table 1 and the optimal sequence is $\rho=\left[J_{6}, J_{2}, J_{3}, J_{1}, J_{5}, J_{7}, J_{4}, J_{8}\right]$, $d_{\mathrm{opt}}=C_{\rho(5)}=\sum_{j=1}^{5}(1+0.5 *(5-j)) p_{\rho(j)}=41.08366$, and $\sum_{i=1}^{n} \omega_{i}\left|L_{\rho(i)}\right|+\omega_{0} d_{\mathrm{opt}}=1421.016$. 
TABLE 1. Values $\theta_{r} p_{i} \max \left\{r^{a_{i}}, b\right\}$.

\begin{tabular}{lcccccccc}
\hline \hline$J_{i} \backslash r$ & $r=1$ & $r=2$ & $r=3$ & $r=4$ & $r=5$ & $r=6$ & $r=7$ & $r=8$ \\
\hline$J_{1}$ & 343.0000 & 278.6514 & 241.9515 & $\mathbf{2 1 4 . 2 4 0 6}$ & 172.2502 & 146.7155 & 113.4000 & 75.60000 \\
$J_{2}$ & 245.0000 & $\mathbf{2 0 1 . 8 1 5 1}$ & 176.6618 & 157.3313 & 127.0607 & 108.6203 & 82.99640 & 54.00000 \\
$J_{3}$ & 294.0000 & 233.9287 & $\mathbf{2 0 0 . 6 6 3 2}$ & 176.1543 & 140.6837 & 122.4000 & 97.20000 & 64.80000 \\
$J_{4}$ & 441.0000 & 353.3337 & 304.3199 & 267.9200 & 214.4494 & 183.6000 & $\mathbf{1 4 5 . 8 0 0 0}$ & 97.20000 \\
$J_{5}$ & 490.0000 & 384.5135 & 327.1705 & 285.5623 & $\mathbf{2 2 8 . 0 0 0 0}$ & 204.0000 & 162.0000 & 108.0000 \\
$J_{6}$ & $\mathbf{1 4 7 . 0 0 0 0}$ & 114.5573 & 97.07875 & 84.48925 & 68.40000 & 61.20000 & 48.60000 & 32.40000 \\
$J_{7}$ & 392.0000 & 316.2590 & 273.4947 & 241.4756 & 193.7144 & $\mathbf{1 6 4 . 6 9 7 3}$ & 129.6000 & 86.40000 \\
$J_{8}$ & 539.0000 & 425.9068 & 363.8631 & 318.5034 & 253.8023 & 224.4000 & 178.2000 & $\mathbf{1 1 8 . 8 0 0 0}$ \\
\hline
\end{tabular}

For a special case: $a_{i}=a$, we have:

$$
\sum_{i=1}^{n} \omega_{i}\left|L_{\rho(i)}\right|+\omega_{0} d_{\mathrm{opt}} / q_{\mathrm{opt}}=\sum_{i=1}^{n} \theta_{i} p_{\rho(i)}^{A}=\sum_{i=1}^{n} \theta_{i} p_{\rho(i)} \max \left\{r^{a}, b\right\} .
$$

Obviously, the minimization of term (5.7) can be obtained by Lemma 3.3 (i.e., $x_{i}=p_{i}, y_{i}=\theta_{i} \max \left\{i^{a}, b\right\}$ ), hence, we have the following result:

Theorem 5.4. The problem $1\left|\mathrm{psd}, \mathrm{CON} / \mathrm{SLK}, d_{\mathrm{opt}} / q_{\mathrm{opt}}, p_{i}^{A}=p_{i} \max \left\{r^{a}, b\right\}\right| \sum_{i=1}^{n} \omega_{i}\left|L_{\rho(i)}\right|+\omega_{0} d_{\mathrm{opt}} / q_{\mathrm{opt}}$ can be solved in $O(n \log n)$ time.

\subsection{Deteriorating jobs}

In this subsection, we introduce deteriorating jobs $[9,30]$ to the scheduling, i.e., the actual processing time of job $J_{i}$ is given by

$$
p_{i}^{A}=p_{i}+c t, i=1, \ldots, n,
$$

where $c \geq 0$ is the deterioration rate, and $t$ is its starting time.

Clearly, Lemmas 3.1, 3.2, 4.1 and 4.2 still hold when deteriorating jobs are introduced. Similar to the above analysis, we have

$$
\sum_{i=1}^{n} \omega_{i}\left|L_{\rho(i)}\right|+\omega_{0} d_{\mathrm{opt}} / q_{\mathrm{opt}}=\sum_{i=1}^{n} \theta_{i} p_{\rho(i)}^{A}=\sum_{i=1}^{n} \Delta_{i} p_{\sigma(i)},
$$

where

$$
\begin{aligned}
& \Delta_{1}=\theta_{1}+c \theta_{2}+c(1+c) \theta_{3}+\ldots+c(1+c)^{n-2} \theta_{n} \\
& \Delta_{2}=\theta_{2}+c \theta_{3}+c(1+c) \theta_{4}+\ldots+c(1+c)^{n-3} \theta_{n} \\
& \Delta_{3}=\theta_{3}+c \theta_{4}+c(1+c) \theta_{5}+\ldots+c(1+c)^{n-4} \theta_{n} \\
& \ldots \\
& \Delta_{n-1}=\theta_{n-1}+c \theta_{n} \\
& \Delta_{n}=\theta_{n},
\end{aligned}
$$

where, for the CON due-date assignment, $\theta_{i}(i=1,2, \ldots, n)$ is given by $(3.4)$; for the SLK due-date assignment, $\theta_{i}(i=1,2, \ldots, n)$ is given by (4.4).

Obviously, the minimization of term (5.9) can be obtained by Lemma 3.3 (i.e., $x_{i}=p_{i}, y_{i}=\Delta_{i}$ ), thus yielding the following result. 
TABLE 2. Scheduling problems with the psd setup times.

\begin{tabular}{lll}
\hline \hline Problem & Complexity & Ref. \\
\hline $1 \mid$ psd, CON, $d_{\text {opt }}\left|\sum_{i=1}^{n} \omega_{i}\right| L_{\rho(i)} \mid+\omega_{0} d_{\mathrm{opt}}$ & $O(n \log n)$ & Theorem 3.5 \\
$1 \mid$ psd, SLK, $q_{\mathrm{opt}}\left|\sum_{i=1}^{n} \omega_{i}\right| L_{\rho(i)} \mid+\omega_{0} q_{\mathrm{opt}}$ & $O(n \log n)$ & Theorem 4.5 \\
$1\left|\mathrm{psd}, \mathrm{CON}, q_{\mathrm{opt}}, p_{i}^{A}=p_{i} \max \left\{r^{a_{i}}, b\right\}\right| \sum_{i=1}^{n} \omega_{i}\left|L_{\rho(i)}\right|+\omega_{0} d_{\mathrm{opt}}$ & $O\left(n^{3}\right)$ & Theorem 5.2 \\
$1\left|\mathrm{psd}, \mathrm{SLK}, q_{\mathrm{opt}}, p_{i}^{A}=p_{i} \max \left\{r^{a_{i}}, b\right\}\right| \sum_{i=1}^{n} \omega_{i}\left|L_{\rho(i)}\right|+\omega_{0} q_{\mathrm{opt}}$ & $O\left(n^{3}\right)$ & Theorem 5.2 \\
$1\left|\mathrm{psd}, \mathrm{CON}, q_{\mathrm{opt}}, p_{i}^{A}=p_{i} \max \left\{r^{a}, b\right\}\right| \sum_{i=1}^{n} \omega_{i}\left|L_{\rho(i)}\right|+\omega_{0} d_{\mathrm{opt}}$ & $O(n \log n)$ & Theorem 5.4 \\
$1\left|\mathrm{psd}, \mathrm{SLK}, q_{\mathrm{opt}}, p_{i}^{A}=p_{i} \max \left\{r^{a}, b\right\}\right| \sum_{i=1}^{n} \omega_{i}\left|L_{\rho(i)}\right|+\omega_{0} q_{\mathrm{opt}}$ & $O(n \log n)$ & Theorem 5.4 \\
$1\left|\mathrm{psd}, \mathrm{CON}, d_{\mathrm{opt}}, p_{i}+c t\right| \sum_{i=1}^{n} \omega_{i}\left|L_{\rho(i)}\right|+\omega_{0} d_{\mathrm{opt}}$ & $O(n \log n)$ & Theorem 5.5 \\
$1\left|\mathrm{psd}, \mathrm{SLK}, q_{\mathrm{opt}}, p_{i}+c t\right| \sum_{i=1}^{n} \omega_{i}\left|L_{\rho(i)}\right|+\omega_{0} q_{\mathrm{opt}}$ & $O(n \log n)$ & Theorem 5.5 \\
\hline
\end{tabular}

Theorem 5.5. The problem $1\left|\mathrm{psd}, \mathrm{CON} / \mathrm{SLK}, d_{\mathrm{opt}} / q_{\mathrm{opt}}, p_{i}^{A}=p_{i}+c t\right| \sum_{i=1}^{n} \omega_{i}\left|L_{\rho(i)}\right|+\omega_{0} d_{\mathrm{opt}} / q_{\mathrm{opt}}$ can be solved in $O(n \log n)$ time.

The following example is only used to illustrate Algorithm 3.4 for the problem $1\left|\mathrm{psd}, \mathrm{CON}, d_{\mathrm{opt}}, p_{i}^{A}=p_{i}+c t\right| \sum_{i=1}^{n} \omega_{i}\left|L_{\rho(i)}\right|+\omega_{0} d_{\mathrm{opt}}$.

Example 5.6. The input data in this example is the same as in Example 3.6 except that $c=0.1$.

By Algorithm 3.4 and Example 3.6, we have $k=5$, and $\theta_{1}=49, \theta_{2}=48, \theta_{3}=46.5, \theta_{4}=44.5$, $\theta_{5}=38, \theta_{6}=34, \theta_{7}=27, \theta_{8}=18$. From (5.10), we have $\Delta_{1}=81.87243, \Delta_{2}=73.52039, \Delta_{3}=65.47308$, $\Delta_{4}=57.70280, \Delta_{5}=46.54800, \Delta_{6}=38.68000, \Delta_{7}=28.80000, \Delta_{8}=18$. From Lemma 3.3, the optimal sequence is $\rho=\left[J_{6}, J_{2}, J_{3}, J_{1}, J_{7}, J_{4}, J_{5}, J_{8}\right], d_{\mathrm{opt}}=C_{\rho(5)}=\sum_{j=1}^{5}(1+0.5 *(5-j)) p_{\rho(j)}^{A}=59.04380$, and $\sum_{i=1}^{n} \omega_{i}\left|L_{\rho(i)}\right|+\omega_{0} d_{\mathrm{opt}}=2616.481$.

\section{Conclusions}

In this paper, we considered single-machine scheduling problems with psd setup times and position-dependent weights. Under the CON and SLK due-date assignment methods, we proved that a non-regular objective function minimization can be solved in $O(n \log n)$ time (see Tab. 2). Further research may study other non-regular objective functions (such as the due-window assignment, Liman et al. [20], Zhang and Wang [39], and Zhang et al. [40]). In addition, multi-machine problems with the psd setup times are also interesting issues.

Acknowledgements. This work was supported by the Natural Science Foundation of Liaoning Province, China (2020-MS$233)$.

\section{REFERENCES}

[1] A. Allahverdi, The third comprehensive survey on scheduling problems with setup times/costs. Eur. J. Oper. Res. 246 (2015) $345-378$.

[2] A. Allahverdi, C.T. Ng, T.C.E. Cheng and M.Y. Kovalyov, A survey of scheduling problems with setup times or costs. Eur. J. Oper. Res. 187 (2008) 985-1032.

[3] D. Biskup, A state-of-the-art review on scheduling with learning effects. Eur. J. Oper. Res. 188 (2008) $315-329$.

[4] D. Biskup and J. Herrmann, Single-machine scheduling against due dates with past-sequence-dependent setup times. Eur. J. Oper. Res. 191 (2008) 587-592.

[5] P. Brucker, Scheduling Algorithms, 3rd edition. Springer, Berlin-Heidelberg (2001).

[6] T.C.E. Cheng, W.-C. Lee and C.-C. Wu, Scheduling problems with deteriorating jobs and learning effects including proportional setup times. Comput. Ind. Eng. 58 (2010) 326-331.

[7] T.C.E. Cheng, W.-C. Lee and C.-C. Wu, Single-machine scheduling with deteriorating jobs and past-sequence-dependent setup times. Appl. Math. Model. 35 (2011) 1861-1867. 
[8] P. Chrétienne, Minimizing the earliness and tardiness cost of a sequence of tasks on a single machine. RAIRO:OR 35 (2001) $165-187$.

[9] S. Gawiejnowicz, Time-Dependent Scheduling. Springer, Berlin-Heidelberg (2008).

[10] V.S. Gordon, J.M. Proth and C.B. Chu, A survey of the state of-the-art of common due date assignment and scheduling research. Eur. J. Oper. Res. 139 (2002) 1-25.

[11] V.S. Gordon, J.M. Proth and C.B. Chu, Due date assignment and scheduling: SLK, TWK and other due date assignment models. Prod. Plan. Control 13 (2002) 117-132.

[12] R.L. Graham, E.L. Lawler, J.K. Lenstra and A.H.G. Rinnooy Kan, Optimization and approximation in deterministic sequencing and scheduling: a survey. Ann. Disc. Math. 5 (1979) 287-326.

[13] G.H. Hardy, J.E. Littlewood and G. Pólya, Inequalities, 2nd edition. Cambridge University Press, Cambridge, UK (1967).

[14] C.-J. Hsu, W.-H. Kuo and D.-L. Yang, Unrelated parallel machine scheduling with past-sequence-dependent setup time and learning effects. Appl. Math. Model. 35 (2011) 1492-1496.

[15] X. Huang, G. Li, Y. Huo and P. Ji, Single machine scheduling with general time-dependent deterioration, position-dependent learning and past sequence-dependent setup times. Optim. Lett. 7 (2013) 1793-1804.

[16] C. Koulamas and G.J. Kyparisis, Single-machine scheduling problems with past-sequence-dependent setup times. Eur. J. Oper. Res. 187 (2008) 1045-1049.

[17] C. Koulamas and G.J. Kyparisis, New results for single-machine scheduling with past-sequence-dependent setup times and due date-related objectives. Eur. J. Oper. Res. 278 (2019) 149-159.

[18] W.-H. Kuo and D.-L. Yang, Single-machine scheduling with past-sequence-dependent setup times and learning effects. Inf. Process. Lett. 102 (2007) 22-26.

[19] W.-H. Kuo, C.-J. Hsu and D.-L. Yang, Some unrelated parallel machine scheduling problems with past-sequence-dependent setup time and learning effects. Comput. Ind. Eng. 61 (2011) 179-183.

[20] S.D. Liman, S.S. Panwalkar and S. Thongmee, Common due window size and location determination in a single machine scheduling problem. J. Oper. Res. Soc. 49 (1998) 1007-1010.

[21] H. Lin, C. He and Y. Lin, Bicriteria scheduling for due date assignment with total weighted tardiness. RAIRO:OR 52 (2018) 359-370.

[22] W. Liu, X. Hu and X.-Y. Wang, Single machine scheduling with slack due dates assignment. Eng. Optim. 49 (2017) $709-717$.

[23] Y.-Y. Lu, G. Li, Y.-B. Wu and P. Ji, Optimal due-date assignment problem with learning effect and resource-dependent processing times. Optim. Lett. 8 (2014) 113-127.

[24] Y.-Y. Lu, F. Teng and Z.-X. Feng, Scheduling jobs with truncated exponential sum-of-logarithm-processing-times based and position-based learning effects. Asia Pac. J. Oper. Res. 32 (2015) 1550026.

[25] V. Mani, P.-C. Chang and S.-H. Chen, Single-machine scheduling with past-sequence-dependent setup times and learning effects: a parametric analysis. Int. J. Syst. Sci. 42 (2011) 2097-2102.

[26] H.M. Soroush, Scheduling in bicriteria single machine systems with past-sequence-dependent setup times and learning effects. J. Oper. Res. Soc. 65 (2014) 1017-1036.

[27] L. Tai, Optimizing batch-processing operations with batch-position-based learning effects. To appear in: RAIRO:OR. DOI: $10.1051 /$ ro/2019108 (2021).

[28] J.-B. Wang, Single-machine scheduling with past-sequence-dependent setup times and time-dependent learning effect. Comput. Ind. Eng. 55 (2008) 584-591.

[29] J.-B. Wang and J.-X. Li, Single machine past-sequence-dependent setup times scheduling with general position-dependent and time-dependent learning effects. Appl. Math. Model. 35 (2011) 1388-1395.

[30] J.-J. Wang and Y.-J. Liu, Single-machine bicriterion group scheduling with deteriorating setup times and job processing times. Appl. Math. Comput. 242 (2014) 309-314.

[31] X.-R. Wang and J.-B. Wang, Scheduling with past-sequence-dependent setup times and learning effects on a single machine. Int. J. Adv. Manuf. Technol. 48 (2010) 739-746.

[32] X.-Y. Wang and J.-J. Wang, Scheduling problems with past-sequence-dependent setup times and general effects of deterioration and learning. Appl. Math. Model. 37 (2013) 4905-4914.

[33] J.-B. Wang, D. Wang, L.-Y. Wang, L. Lin, N. Yin and W.-W. Wang, Single machine scheduling with exponential time-dependent learning effect and past-sequence-dependent setup times. Comput. Math. App. 57 (2009) 9-16.

[34] X.-R. Wang, J. Jin, J.-B. Wang and P. Ji, Single machine scheduling with truncated job-dependent learning effect. Optim. Lett. 8 (2014) 669-677.

[35] J.-B. Wang, X.-N. Geng, L. Liu, J.-J. Wang and Y.-Y. Lu, Single machine CON/SLK due date assignment scheduling with controllable processing time and job-dependent learning effects. Comput. J. 61 (2018) 1329-1337.

[36] J.-B. Wang, F. Liu and J.-J. Wang, Research on $m$-machine flow shop scheduling with truncated learning effects. Int. Trans. Oper. Res. 26 (2019) 1135-1151.

[37] J.-B. Wang, M. Gao, J.-J. Wang, L. Liu and H. He, Scheduling with a position-weighted learning effect and job release dates. Eng. Optim. 52 (2020) 1475-1493.

[38] X. Xiong, D. Wang, T.C.E. Cheng, C. Wu and Y. Yin, Single-machine scheduling and common due date assignment with potential machine disruption. Int. J. Prod. Res. 56 (2018) 1345-1360.

[39] X. Zhang and Y. Wang, Single-machine scheduling CON/SLK due window assignment problems with sum-of-processed time based learning effect. Appl. Math. Comput. 250 (2015) 628-635.

[40] X. Zhang, W. Lin, W. Wu and C. Wu, Single-machine common/slack due window assignment problems with linear decreasing processing times. Eng. Optim. 49 (2017) 1388-1400. 